\title{
DIGITAL TECHNOLOGY Older people and technology: time to smarten up our act
}

\author{
Authors: Hanad Ahmed, ${ }^{\mathrm{A}}$ Iqraa Haq, ${ }^{\mathrm{B}}$ Ammar Rahman, ${ }^{\mathrm{B}}$ Emma Tonner, ${ }^{\mathrm{C}}$ Rami Abbass, ${ }^{\mathrm{B}}$ Faraz Sharif, \\ Shad Asinger ${ }^{B}$ and Magda Sbai ${ }^{D}$
}

The NHS faces challenges today that it was not designed to tackle at its conception in 1948. The UK demographic has changed considerably with higher life expectancy and 'an ageing population'.

Keeping this demographic healthy through prevention and management of age-related degeneration is crucial to their independence and improving resource utilisation. The Department of Health and Social Care's agenda for digital transformation of the NHS is facilitating a move towards preventative healthcare and greater community care, which will likely be supported by virtual healthcare delivery models. Despite views on digital illiteracy in the older population, this demographic may stand to benefit the most.

Research has shown that the older demographic adopts technology in line with the technology acceptance model if their needs are carefully considered. Executed successfully, the deployment of virtual healthcare could save transformational costs to the NHS and support better quality of life for the senior members of society.

This is particularly relevant in the current COVID-19 pandemic with patients facing challenges in accessing outpatient appointments. With many hospitals kickstarting virtual outpatient clinics to ensure continuity of care during a time of social isolation; we await to see the ingenuities that arise from the current pandemic.

KEYWORDS: virtual care, older population, technology

DOI: $10.7861 /$ fhj.2020-0015

\section{Ageing population}

Our nation remains unwavering in the commitment to universal healthcare, irrespective of age, health, race, social status or ability to pay. To high quality care for all. - NHS Five Year Forward View, 2014.

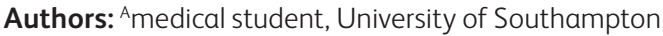
Southampton UK and Imperial College London, London, UK; ${ }^{B}$ medical student, Imperial College London, London, UK; Cmedical student, University of Leeds, Leeds, UK and Imperial College London, London, UK; ' ${ }^{2}$ consultant physician and geriatrician, Guy's and St Thomas' NHS Foundation Trust, London, UK
The NHS faces challenges today that it was not designed to tackle at its inception in 1948. The demographic of the country has changed, with improvements in healthcare, education and technology resulting in higher life expectancy and what is popularly referred to as 'an ageing population'. While the proportion of the population living past the age of 65 has and is further set to rise in coming years, the current healthcare system is struggling to keep pace. The older population, largely defined as over 65 although this definition differs according to source used, play a central role in society. ${ }^{2}$ As a nation, it is our responsibility to ensure that they stay socially engaged and retain their independence. This is even more important in the face of the social care crisis, with many older people not receiving the care they need due to funding constraints. ${ }^{3}$ Keeping this demographic healthy through prevention and effective management of agerelated degeneration is crucial if we are to maximise independence and improve resource utilisation.

We must also consider and challenge the bias that exists within society and our healthcare system about older age. The over 65 demographic is more active than ever before, representing a significant part of the workforce. Better health also means people are increasingly expecting to lead more active retirement lives at a much older age than would have been possible in the past. The uptake of technology within society has also extended far beyond traditional target groups, and now forms a key component of al lives, irrespective of age. It is therefore important to modernise and redefine what it means to be 'old' and to tailor technology to all age groups in order to leverage the true potential of digital health in the NHS.

The newly formed NHSX is spearheading an agenda for digital transformation of the NHS as outlined in The NHS Long Term Plan. 4.5 Some of the aims include leveraging technology to facilitate a move towards preventative healthcare and a greater level of care in the community. Although the technology modalities being used to achieve this goal are vast, one of the most relevant for chronic disease management and community care is the virtual healthcare model.

\section{What is virtual healthcare?}

The promise of technology and innovation in the form of virtual healthcare is significant. However, the term virtual healthcare must first be defined. This poses a challenge as the definition of 'virtual healthcare' remains broad and varies within the literature. 
The Women's College Hospital Institute for Health Systems Solutions and Virtual Care provides a useful definition for understanding which technologies fall into this category; it defines virtual healthcare as: ${ }^{6}$

... any interaction between patients and/or members of their circle of care, occurring remotely, using any forms of communication or information technologies, with the aim of facilitating or maximizing the quality and effectiveness of patient care.

Although not interchangeable, it is not uncommon for literature to further refer to virtual care using two other terms: telehealth and telecare. Telehealth can be defined as the use of technology to improve healthcare delivery in both clinical and non-clinical services such as medical education and administration. The clinical component of telehealth is called telemedicine and is widely cited in literature. It uses technology to engage patients remotely and manages medical care without in-person visits. These technologies include video consultations, digital health in the form of health apps, telephone consultations, electronic health and secure messaging.?

In contrast, telecare is centred around using technology to automatically monitor patients remotely, keeping care in the community and minimising readmissions to hospital through early intervention. ${ }^{8}$ The use of both these models of virtual care is widespread in the USA and other insurance-based healthcare systems that focus on reducing costs to the hospital by keeping healthcare delivery in the community. ${ }^{9}$

Countries like Australia have also adopted technology that facilitates virtual care due to the vast geographic distances that patients and clinicians struggle to cover logistically, improving care delivery and saving both time and cost. The widespread adoption of virtual care in the UK, however, has been slow. In response to the ageing population and the coinciding rise in the proportion of people living with long-term conditions, the UK government commissioned the Whole System Demonstrator (WSD) programme, the largest randomised controlled trial (RCT) of telehealth and telecare in the world..$^{10}$ The RCT evaluated the uses of telehealth and telecare pilots in Kent, Cornwall and Newham..$^{10}$ Although the early findings of the RCT showed mixed cost-effectiveness, it also showed that telehealth delivered a reduction in emergency department visits, a drastic $45 \%$ reduction in mortality rates, as well as an $8 \%$ reduction in tariff costs. Despite these benefits, a widespread concern that continues to persist is that these technology modalities and care models will not benefit those that need it most (ie the over 65 age group) due to technology illiteracy.

\section{The older population and technology}

Local service leaders are already innovating, implementing and transforming services for older people. What we need now is to disseminate their experiences and the lessons learnt so that those models can be adopted more widely, making the rest as good as the best. - Kings Fund, 2014.11

There has been significant growth in the usage of technology by the older population. An Office for National Statistics report demonstrated that the largest relative increase in daily computer usage occurred in those aged over 65 between 2006 and $2014 .{ }^{12}$ Additionally, with greater emphasis placed on involving older populations in participatory design, technology can effectively meet their needs and capabilities, encouraging greater engagement with technological devices. ${ }^{13}$ Wilkinson et al demonstrated the ease with which older adults can be involved in product design; highlighting the resultant improvement in usability and accessibility of their technology to older age groups as their input would allow users to 'operate at a more unconscious, automatic and skill-based level. ${ }^{14}$ Yet, there is evidence to suggest that older populations often lag in adopting newer technology, relative to other age groups, with many assuming that they are generally technologically illiterate. ${ }^{T}$ The reasons for slower adoption are explored by a number of studies. Renaud et al found that participants often fell into two distinct groups: those who had mastered the 'beast' and those who were bemused by it. ${ }^{15}$ The former were more adventurous and used more than just the basic communication functionalities of their phones, whereas the latter appeared to own the phone merely because it was given to them. The authors go on to discuss the senior technology acceptance and adoption model (STAM), a conceptual model that outlines the phases of technology adoption. The STAM model suggests a three-step process starting with the 'objectification' phase in which the user establishes the utility of the device, and is influenced socially (eg by family members) and by the perceived usefulness of the device. The 'incorporation' phase is when the user attempts to incorporate the device into their daily life, this mostly consists of experimentation and exploration. How successful they are incorporating the device is influenced by how useful they find the device, the ease of learning and other 'facilitating factors' such as device costs and maintenance. The final phase is the 'conversion' phase and determines whether the user accepts or rejects the technology.

Vaportzis et al and Galambos et al both showed that the cost of purchasing the technological devices and the subsequent lack of information on how to use the devices are some of the reasons for slower adoption among the older population.16,17 The older population are also identified as having particular difficulties in engaging with technology due to the cognitive and visual decline associated with ageing as well as the decline in fine motor skills and dexterity.

Hanson et al provide a different perspective. ${ }^{18}$ The authors discuss the wisdom of older people and their societal concerns as an important factor in the resistance to technology adoption. They highlight some of the perceived negative consequences of technology on face-to-face communication and a concern around depersonalising services.

In contrast to these findings, a report evaluating technology trends by Deloitte showed that $77 \%$ of the over 55 age group use smartphones, with the strongest growth in smartphone ownership in this age group. ${ }^{19}$ With the increasing public expectation of living a more active retirement life, it can be argued that technology penetration into this age group will continue to rise as it has over the past 6 years, making mobile health and virtual care platforms a promising opportunity for the older patient and their clinicians. Nationwide efforts to further increase uptake of technological devices are already underway, with programmes such as the One Digital programme by Age UK, demonstrating the success of educating the older generation so that they are subsequently able to engage with technology. It is important to remember that in order to promote the adoption of any innovation to any target group, it must be user-friendly, addressing the needs of the consumer to provide a tangible benefit. ${ }^{20,21}$ Although the 
older population may arguably be more reluctant to change in comparison to their younger counterparts, numerous studies have shown that older people adopt new technology in line with the technology acceptance model. ${ }^{20,22}$ The model considers variables such as perceived ease of use, perceived benefit, attitudes and behaviours toward the modality and ease of engagement with the technology. ${ }^{20,22}$ An important factor to also consider is that perceived ease of use has also been suggested to influence perceived usefulness of the technology modality, highlighting the importance of the associations between different factors. ${ }^{23}$ The model is illustrated by Rahimi et al in Applied Clinical Informatics. ${ }^{24}$

\section{Public health crisis and virtual care}

Having discussed the use of virtual care and the factors supporting its widespread acceptance among the older demographic, it is also important to consider the additional potential of virtual care in times of public health crisis. As the world is gripped by the COVID-19 pandemic, the use of virtual care in the management of patients, particularly those that are older with chronic conditions, becomes even more topical considering the higher risk of mortality from COVID-19. Government legislation regarding social isolation and quarantine has also made it particularly challenging for these patient groups to access healthcare services without risking exposure.

Many trusts have therefore changed their approach by increasing uptake of existing virtual care services and rolling out widespread virtual outpatient clinics in efforts to meet the needs of chronic disease patients while from the safety of their own home. This would reduce the potential for more COVID-19 cases, in turn decreasing the chances of NHS capacity being breached.

There are a number of examples illustrating the potential of virtual care during the COVID-19 pandemic. One notable example comes from University Hospitals of Leicester NHS Trust which saw video consultations making up 10\% of consultations by May 2020 to manage a wide range of patients, from those with Parkinson's disease receiving online therapy to diabetic patients being taught how to administer insulin. ${ }^{25}$

A number of healthcare providers in the USA have also adopted virtual care via electronic intensive care unit monitoring to allow for the remote assessment of 60-100 patients in multiple hospitals. ${ }^{26}$ Other providers promoted the uptake of community mobile integrated healthcare programmes which have permitted treatment at home to curb the influx of patients to the emergency department. $^{26}$

Whether or not these changes will last beyond the COVID-19 outbreak is uncertain. However, we hope that the ingenuities adopted during this crisis will have lasting positive effects on health services and the patient groups that rely on them.

\section{Conclusion}

By meeting the relatively higher threshold of perceived benefit in the older age group, technology adoption can still be achieved. It is therefore important to appreciate the promise of technological solutions as key enablers for services accessed by those over 65 .

The design of the technology, however, must suit the needs of this population group to facilitate adoption in line with the technology acceptance model. Future studies should be aimed at gathering the views of this demographic to gain more accurate insight. The older generation arguably stand the most to benefit from virtual care models, particularly in times such as these. Making these care models accessible could enable better selfmanagement, improved independence and safety in the setting of social isolation during disease outbreaks. In turn, this could mean that significant cost savings are made in primary and community care as well as acute care services, creating a sustainable transformation of the NHS.

\section{References}

1 NHS. Five Year Forward View. NHS, 2014.

2 NHS England. Improving care for older people. NHS, 2017. www. england.nhs.uk/ourwork/clinical-policy/older-people/improvingcare-for-older-people [Accessed 15 August 2020].

3 Fund The Kings. Adult social care. The Kings Fund, 2019. www. kingsfund.org.uk/topics/adult-social-care [Accessed 15 August 2020].

4 NHSX. Who we are. NHS, 2020. www.nhsx.nhs.uk/about-us/whowe-are [Accessed 15 August 2020].

5 England NHS. The NHS Long Term Plan. NHS, 2019. www.longtermplan.nhs.uk [Accessed 15 August 2020].

6 Jamieson T, Wallace R, Armstrong K et al. Virtual care: A framework for a patient-centric system. Women's College Hospital Institute for Health Systems Solutions and Virtual Care, 2015. www. womenscollegehospital.ca/assets/pdf/wihv/WIHV_VirtualHealth Symposium.pdf [Accessed 15 August 2020].

7 McGrail KM, Ahuja MA, Leaver CA. Virtual visits and patientcentered care: results of a patient survey and observational study. J Med Internet Res 2017;19:e177

8 McCue MJ, Mazmanian PE, Hampton C et al. The case of Powhatan correctional center/Virginia department of corrections and Virginia commonwealth university/Medical college of Virginia. Telemed J 1997:3:11-7.

9 Gill M. A National telehealth strategy for Australia-for discussion. Australian National Consultative Committee on Electronic Health, 2011.

10 Newman SP, Bardsley M, Barlow ] et al. The whole system demonstrator programme. Department of Health, 2013.

11 Oliver D, Foot C, Humphries R. Making our health and care systems fit for an ageing population. The King's Fund, 2014.

12 Office for National Statistics. Internet access - households and individuals, Great Britain: 2014. ONS, 2014.

13 Hakobyan L, Lumsden J, O'Sullivan D. Participatory design: how to engage older adults in participatory design activities. Int J Mob Hum Comput Interact 2015;7:78-92.

14 Wilkinson C, Cornish K. An overview of participatory design applied to physical and digital product interaction for older people. Multimodal Technologies and Interact 2018;2:79.

15 Renaud K, van Biljon J. Predicting technology acceptance and adoption by the elderly. Proceedings of the 2008 annual research conference of the South African Institute of Computer Scientists and Information Technologists on IT research in developing countries riding the wave of technology 2008:210-9.

16 Vaportzis E, Giatsi Clausen M, Gow AJ. Older adults perceptions of technology and barriers to interacting with tablet computers: a focus group study. Front Psychol 2017;8:1687.

17 Galambos C, Rantz M, Back J et al. Older adults' perceptions and preferences of a fall risk assessment system: Exploring stages of acceptance model. Comput Inform Nurs 2017;35:331-7.

18 Knowles B, Hanson VL. The wisdom of older technology (non-) users. Communications of the ACM 2018;61:72-7.

19 Deloitte. Global Mobile Consumer Survey 2018: The UK cut. Deloitte, 2018. www.deloitte.co.uk/mobileuk2018 [Accessed 15 August 2020].

20 Chen K, Chan AHS. A review of technology acceptance by older adults. Gerontechnology 2011;10:1-12. 
21 Venkatesh V, Davis FD. A theoretical extension of the technology acceptance model: Four longitudinal field studies. Management Science 2000:46:186-204.

22 Heinz M, Martin P, Margrett JA et al. Perceptions of technology among older adults. J Gerontol Nurs 2013;39:42-51.

23 Davis FD. Perceived usefulness, perceived ease of use, and user acceptance of information technology. MIS Quarterly 1989;13:319.

24 Rahimi B, Nadri H, Afshar HL, Timpka T. A systematic review of the technology acceptance model in health informatics. Appl Clin Inform 2018;9:604-34.
25 University Hospitals of Leicester NHS Trust. Virtual NHS consultations hailed a success during coronavirus pandemic. NHS, 2020.

26 Hollander JE, Carr BG. Virtually perfect? Telemedicine for COVID-19. N Engl J Med 2020;382:1679-81.

Address for correspondence: Hanad Ahmed, University of Southampton, Faculty of Medicine, 12 University Road, Building 85, Southampton S017 1BJ, UK.

Email: Ha1g14@soton.ac.uk 